\title{
Optimal post-selection inference for sparse signals: a nonparametric empirical Bayes approach
}

\author{
Spencer Woody* \\ Department of Integrative Biology, \\ The University of Texas at Austin \\ and \\ Oscar Hernán Madrid-Padilla \\ Department of Statistics, The University of California, Los Angeles \\ and \\ James G. Scott \\ Department of Information, Risk, and Operations Management, \\ The University of Texas at Austin
}

November 17, 2020

\begin{abstract}
Many recently developed Bayesian methods have focused on sparse signal detection. However, much less work has been done addressing the natural follow-up question: how to make valid inferences for the magnitude of those signals after selection. Ordinary Bayesian credible intervals suffer from selection bias, as do ordinary frequentist confidence intervals, owing to the fact that the target of inference is chosen adaptively. Existing Bayesian approaches for correcting this bias produce credible intervals with poor frequentist properties, while existing frequentist approaches require sacrificing the benefits of shrinkage typical in Bayesian methods, resulting in confidence intervals that are needlessly wide. We address this gap by proposing a nonparametric empirical Bayes approach for constructing optimal selection-adjusted confidence sets. Our method produces confidence sets that are as short as possible on average, while both adjusting for selection and maintaining exact frequentist coverage uniformly over the parameter space. Our main theoretical result establishes an important consistency property of our procedure: that under mild conditions, it asymptotically converges to the results of an oracle-Bayes analysis in which the prior distribution of signal sizes is known exactly. Across a series of examples, the method outperforms existing frequentist techniques for post-selection inference, producing confidence sets that are notably shorter but with the same coverage guarantee.
\end{abstract}

Keywords: Biased test; Coverage; Post-selection inference; Selection bias; Shrinkage

${ }^{*}$ Corresponding author. Email to spencer. woody@utexas.edu 


\section{Introduction}

This paper proposes a nonparametric empirical Bayes approach to post-selection inference with an exact coverage guarantee. Our framework and theoretical results here are both very general, but we focus primarily on the commonly encountered setting of inference for a sparse vector of Gaussian means, where $y_{i} \sim N\left(\theta_{i}, \sigma^{2}\right)$, and where most $\theta_{i}$ are zero or negligibly small. This model, although simple, is ubiquitous in modern statistical practice.

Much methodological work in this setting has focused on the question of how to find significantly nonzero $\theta_{i}$ 's. Many Bayesian approaches have been based on the two-groups model (e.g. Efron et al., 2001; Scott and Berger, 2006; Efron, 2008), where the prior is a mixture of a point mass at zero and some distribution of nonzero signals, or on global-local shrinkage priors (Polson and Scott, 2011) such as the horseshoe (Carvalho et al. 2010) or the Bayesian lasso (Park and Casella, 2008). But regardless of the particular prior used, an important question that is not adequately addressed in this literature is how to quantify the magnitude of those signals selected for inference after the detection procedure is applied. Previous scholars have differed on whether this poses a problem if one adopts a pure Bayesian perspective (c.f. Dawid, 1994; Yekutieli. 2012). However, even if one does correctly adjust posterior inference for selection when necessary, using posterior credible interavls certainly presents a very stark problem from a frequentist perspective. It is widely known that Bayesian credible sets in general are not valid frequentist confidence sets, except under very specific "matching” priors (e.g. Ghosh, 2011), but rather only retain coverage on average with respect to the prior. Furthermore, in post-selection settings, the departure from nominal coverage is much more severe than is commonly appreciated. For example, we present in $\$ 2.3$ a simple case where, under a plausible selection mechanism, the coverage of $90 \%$ Bayesian credible sets is actually less than $50 \%$ for very strong signals.

There is, of course, an active line of recent work on purely frequentist solutions to the postselection inference problem (e.g. Benjamini and Yekutieli, 2005; Lee et al., 2016; Reid et al., 2014). However, these adjustment procedures do not borrow information across components of the $\theta$ vector, and as a result, they produce confidence sets that are needlessly wide. This is particularly problematic in post-selection inference because selective confidence intervals are necessarily wider than their ordinary (non-selective) counterparts. There have been several attempts to create shorter selection-adjusted frequentist intervals (Zhong and Prentice, 2008; Weinstein 
et al., 2013), but none of these methods have explicitly incorporated Bayesian ideas to improve the efficiency of inference.

Thus there is a major unmet need for inferential procedures that: (i) correctly adjust for the effects of selection; (ii) maintain valid frequentist coverage, uniformly across the whole parameter space; and (iii) produce confidence sets that are as short as possible. The approach we propose here has all three of these desirable features. As we will illustrate, while our procedure is frequentist in nature, its efficiency gains arise from Bayesian thinking: that is, from positing the existence of a prior that describes the sparsity pattern in the data set, and then estimating that prior nonparametrically using modern empirical Bayes tools.

\section{Efficient selection-adjusted inference}

\subsection{Exchangeable means with known prior}

In this section we describe our procedure under the simplifying assumption that the signals $\theta_{i}$ arise from a known prior distribution $\pi(\theta)$. In $\$ 3$, we will come to the core of our proposal, when we describe a method for the more realistic setting of unknown $\pi(\theta)$.

Our work builds off the "frequentist assisted by Bayes" (FAB) framework, which was articulated in its earliest form by Pratt (1963) and substantially extended by Yu and Hoff $(2018)$. This technique was originally proposed for constructing confidence intervals for group-level means in hierarchical normal models, and has also been extended to constructing confidence intervals for coefficients in a linear regression by Hoff and $\mathrm{Yu}$ (2019). Here we generalize the FAB procedure to produce Bayes-optimal confidence sets under post-selection inference. We call this procedure selection-adjusted FAB (saFAB).

Suppose we observe $y_{1}, \ldots, y_{n}$ independently from the parametric model $y_{i} \sim f\left(y_{i} ; \theta_{i}\right)$, and we consider inference only for units $i$ such that $y_{i} \in S$ for some region $S \subset \mathbb{R}$, which we refer to as the selection region or selection event. Conditional on this selection event, $y$ has the truncated density function

$$
f_{S}(y ; \theta)=f(y ; \theta) \cdot \mathbf{1}(y \in S) / \int_{S} f(y ; \theta) \mathrm{d} y
$$


with cumulative density function $F_{S}(y ; \theta)$ and generalized quantile function $F_{S}^{-1}(p ; \theta)=\inf _{y}\{y$ : $\left.F_{S}(y ; \theta) \geq p\right\}$. Using (1), one may construct confidence sets that condition on the selection event $y \in S$ via the well-known procedure of inverting a family of $\alpha$-level tests of point null hypotheses of the form $H_{0}: \theta=\theta_{0}$ for all $\theta_{0} \in \mathbb{R}$. The key decision that arises in this procedure, and that we will discuss in detail below, is how to construct this family of tests.

We first remark that the standard choice of an equal-tailed test is the universally most powerful unbiased (UMPU) test and forms the basis of the method proposed by Fithian et al. (2014). In particular, suppose that we condition on $y \in S$, so that $y \sim f_{S}(y ; \theta)$. Then the UMPU test of $H_{0}: \theta=\theta_{0}$ has acceptance region

$$
A^{S}\left(\theta_{0}\right)=\left\{y: F_{S}^{-1}\left(\alpha / 2 ; \theta_{0}\right) \leq y \leq F_{S}^{-1}\left(1-\alpha / 2 ; \theta_{0}\right)\right\} .
$$

As shown by Fithian et al. (2014), inverting this family of acceptance regions gives the $(1-\alpha)$ level uniformally most accurate unbiased (UMAU) selection-adjusted confidence interval, of the form $C^{S}(y)=\left\{\theta: y \in A^{S}(\theta)\right\}$. It is clear this interval will cover the true $\theta$ with the correct probability, conditional on the selection event $y \in S$, because $\operatorname{Pr}_{\theta}\left(\theta \in C^{S}(y) \mid y \in S\right)=$ $\operatorname{Pr}_{\theta}\left(y \in A^{S}(\theta)\right)=1-\alpha$.

However, following the logic that both Pratt (1963) and Yu and Hoff (2018) apply to the task of ordinary (non-selection-adjusted) inference, we need not restrict ourselves to confidence sets based on the unbiased test using equal-tail probability regions in $F_{S}(y ; \theta)$. In fact, we may generally choose a biased test with acceptance region.

$$
A_{w}^{S}\left(\theta_{0}\right)=\left\{y: F_{S}^{-1}\left(\alpha w ; \theta_{0}\right) \leq y \leq F_{S}^{-1}\left(\alpha w+1-\alpha ; \theta_{0}\right)\right\},
$$

where $w \in[0,1]$ controls how $\alpha$, the total probability mass that falls outside the acceptance region, is split between the two tails of $f_{S}(y ; \theta)$. This leads to confidence sets of the form $C_{w}^{S}(y)=\left\{\theta: y \in A_{w}^{S}(\theta)\right\}$, which will retain nominal coverage by construction. We note that the choice $w=0.5$ recovers the equal-tailed UMPU test and thus the confidence sets from Fithian et al. (2014); any other choice will put different probabilities in the left versus right tail. In fact, we are free to choose a different value of $w$ for each $\theta_{0}$ in Eq. (2), and may tune these choices to reflect our prior knowledge. Specifically, we will find a form of $w$ to give the shortest confidence 
sets for $\theta$, in expectation under a prior $\pi(\theta)$. The result will be a function $w: \mathbb{R} \rightarrow[0,1]$ that gives a different optimal $w$ for each $\theta$, yielding a family of acceptance regions $A_{w(\theta)}^{S}$ and confidence set procedure $C_{w(\theta)}^{S}$. Following Yu and Hoff $(2018)$, we call $w(\theta)$ the spending function, as it dictates how the acceptance region allocates, or spends, the Type I error rate $\alpha$.

The Bayes-optimal spending function is found as follows. First, following Pratt (1961) and Yu and Hoff (2018), define the frequentist risk $R(\theta ; w)$ of a confidence-set procedure for $\theta$ to be the expected Lebesgue measure of that confidence region under $f_{S}(y ; \theta)$. That is,

$$
R(\theta ; w)=\iint \mathbf{1}\left(y \in A_{w}^{S}(\tilde{\theta})\right) f_{S}(y ; \theta) \mathrm{d} \tilde{\theta} \mathrm{d} y
$$

Then, upon introducing a prior $\theta \sim \pi(\theta)$, we may compute the Bayes loss of a confidence set $C_{w}^{S}$ procedure as

$$
\begin{aligned}
L(\pi, w) & =\int R(\theta ; w) \pi(\theta) \mathrm{d} \theta=\int\left[\iint \mathbf{1}\left(y \in A_{w}^{S}(\tilde{\theta})\right) f_{S}(y ; \theta) \mathrm{d} \tilde{\theta} \mathrm{d} y\right] \pi(\theta) \mathrm{d} \theta \\
& =\int\left[\iint \mathbf{1}\left(y \in A_{w}^{S}(\tilde{\theta})\right) f_{S}(y ; \theta) \pi(\theta) \mathrm{d} y \mathrm{~d} \theta\right] \mathrm{d} \tilde{\theta}=\int \operatorname{Pr}\left(Y \in A_{w}^{S}(\tilde{\theta})\right) \mathrm{d} \tilde{\theta} .
\end{aligned}
$$

The final integrand in Eq. (3) is the marginal probability that $y$ falls in the acceptance region, integrating out $\theta$ under the prior. Intuitively, confidence procedures that yield shorter intervals, on average under the marginal distribution of selected observations, have smaller Bayes loss. Let $m_{S}(y)$ denote the density of this marginal distribution, whose form we discuss in the following subsection, with corresponding cumulative density function $M_{S}(y)$. We may rewrite the integrand in (3) as

$$
H(w ; \theta) \equiv \operatorname{Pr}\left(Y \in A_{w}^{S}(\theta)\right)=M_{S}\left[F_{S}^{-1}(\alpha w+1-\alpha ; \theta)\right]-M_{S}\left[F_{S}^{-1}(\alpha w ; \theta)\right]
$$

This defines an objective whose minimization gives the Bayes-optimal spending function,

$$
w^{\star}(\theta)=\arg \min _{w \in[0,1]} H(w ; \theta)
$$

Generally, there is no closed-form solution to solve this optimization problem. Therefore we implement a numerical approach whereby the spending function is approximated pointwise by 
solving the minimization problem along a grid of $\theta$ values. After the optimal spending function is calculated, the Bayes-optimal confidence set for selected observations is obtained by inverting the resulting acceptance region, $C_{w^{\star}(\theta)}^{S}=\left\{\theta: y \in A_{w^{\star}(\theta)}^{S}\right\}$.

We note that $w(\theta)$ must be a monotonic function to ensure that the produced confidence sets are intervals. This is sometimes not the case, as in the toy example presented later in this section. Thus, the returned confidence set will occasionally be a set of disjoint intervals, in which case the size of the set is equal to the Lebesque measure. However, this rarely happens in all our applications. If such a confidence set is produced, one may create an interval by taking the minimum and maximum of the set, and then the size of this interval would be given by the difference. Such a procedure will produce confidence intervals with conservative coverage and which will often still be more efficient than the UMAU intervals.

\subsection{Influence of the selection mechanism on the spending function}

We observe from Eq. (4) that calculating the optimal spending function requires knowing two parts of the overall model: the truncated sampling model $f_{S}(y ; \theta)$ and the marginal density of selected signals $m_{S}(y)$. As we shall see, this marginal density depends both on the prior $\pi(\theta)$ as well as the specific mechanism by which selection occurs.

A very useful distinction between two different types of selection mechanisms-joint selection versus conditional selection-was drawn by Yekutieli (2012). This distinction is best explained by example. To understand joint selection, imagine a genomics study that seeks to understand the differences in gene expression across two experimental conditions. Suppose the data arise as follows. For each gene $i=1, \ldots, N$ : (i) draw $\theta_{i} \sim \pi(\theta)$, representing a true effect size for gene $i$; (ii) observe data $y_{i} \sim N\left(\theta_{i}, 1\right)$, representing the observed effect size for gene $i$, (iii) select the $\left(y_{i}, \theta_{i}\right)$ pairs where $y_{i} \in S$ (e.g. $\left|y_{i}\right|>2$ ), and perform inference only for those selected $\theta_{i}$ 's. Under such a scenario, the selected $y$ and $\theta$ have the joint distribution

$$
p_{S}^{(J)}(\theta, y)=\pi(\theta) \cdot f(y ; \theta) \cdot \mathbf{1}(y \in S) / \operatorname{Pr}(S),
$$

where $\operatorname{Pr}(S)=\iint_{S} f(y ; \theta) \pi(\theta) \mathrm{d} y \mathrm{~d} \theta$ is the marginal probability of selection. In this case, the marginal density of selected signals is proportional to the ordinary marginal density $m(y)=$ 
$\int f(y ; \theta) \pi(\theta) \mathrm{d} \theta$, truncated to $S$ :

$$
m_{S}^{(J)}(y)=\int \mathbf{1}(y \in S) \pi(\theta) f(y ; \theta) / \operatorname{Pr}(S) \mathrm{d} \theta=m(y) \cdot \mathbf{1}(y \in S) / \operatorname{Pr}(S) .
$$

We use the term "joint selection" rather than Yekutieli's term "random parameter selection" to describe such a setting, to emphasize that the selection mechanism applies to $\left(y_{i}, \theta_{i}\right)$ pairs jointly.

Now turning to conditional selection, imagine a scientific field with many open questions $(i=1,2, \ldots)$, where journals publish results only for observed effect sizes $y \in S$. For each open question $i$, published results are generated as follows: (i) sample $\theta_{i} \sim \pi(\theta)$, representing the true effect size for question $i$; (ii) many labs $(k=1,2, \ldots)$ observe $y_{i}^{(k)} \sim N\left(\theta_{i}, \sigma^{2}\right)$; (iii) the first lab that observes $y_{i}^{(k)} \in S$ publishes its results. Now let $y_{i}$ denote the published estimate for $\theta_{i}$; by construction, this is a sample from $N\left(\theta_{i}, \sigma^{2}\right)$ truncated to $S$, taken by rejection sampling. We refer to this mechanism as "conditional selection", rather than Yekutieli's term of "fixed parameter" selection, to emphasize that the selection mechanism applies to draws for $y_{i}$, conditional upon a specific value of $\theta_{i}$, which is itself assumed to be a random draw from a prior.

Under conditional selection, the joint distribution of $(\theta, y)$ for selection signals is now

$$
p_{S}^{(C)}(\theta, y)=\pi(\theta) \cdot f(y ; \theta) \cdot \mathbf{1}(y \in S) / \operatorname{Pr}(S \mid \theta),
$$

where $\operatorname{Pr}(S \mid \theta)=\int_{S} f(y ; \theta) \mathrm{d} \theta$ is the probability of selection conditional on $\theta$. This implies that the marginal density of the data is

$$
m_{S}^{(C)}(y)=\mathbf{1}(y \in S) \int \pi(\theta) f(y \mid \theta) / \operatorname{Pr}(S \mid \theta) \mathrm{d} \theta .
$$

Unlike in the case of joint selection, this marginal is no longer equivalent to the usual marginal density truncated to $S$, because of the term $\operatorname{Pr}(S \mid \theta)$ appearing inside the integral over $\theta$.

Thus we see that the optimal spending function depends on four factors: the parametric model $f(y ; \theta)$, the selection set $S$, the prior $\pi(\theta)$, and the selection mechanism itself. 


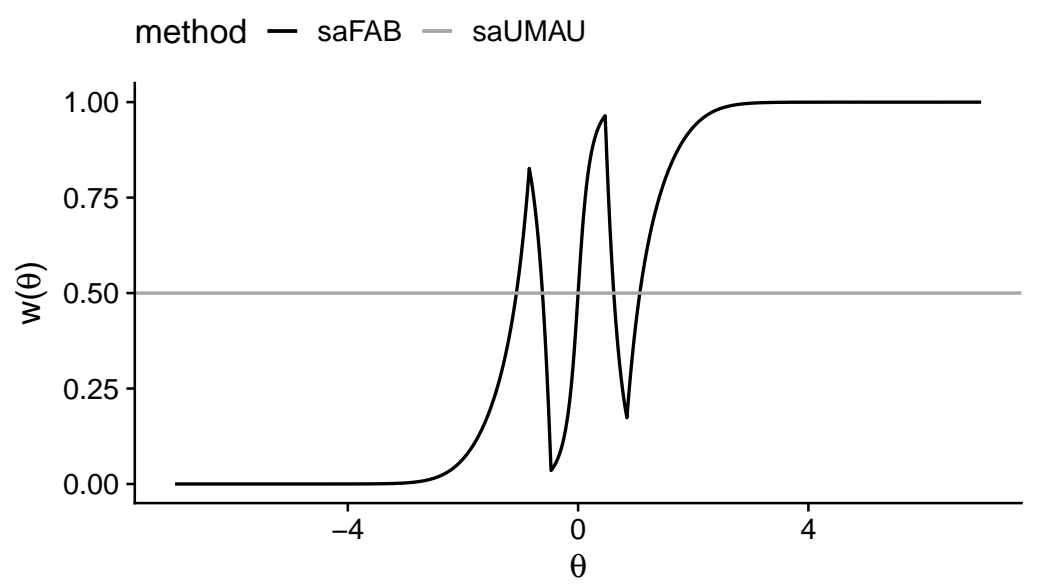

Figure 1: Spending function from the toy example, which determines the degree of bias in acceptance region calculations as a function of $\theta$. Inversion of these acceptance regions gives the Bayes-optimal confidence sets. The spending function depends on (i) the sampling distribution of observations, (ii) the selection set, (iii) the selection mechanism, and (iv) the prior for signals.

\subsection{Toy example}

To demonstrate the mechanics and advantages of our method, we first show how it performs on a toy example where $\theta$ follows the two-groups model,

$$
\pi(\theta)=p \cdot N\left(\theta ; 0, \tau^{2}\right)+(1-p) \cdot \delta_{0}(\theta)
$$

a mixture of a point mass at 0 and a zero-centered Gaussian with variance $\tau^{2}$. For this example we set $p=0.1$ and $\tau^{2}=3$. We generate $n=10,000$ samples of $\theta_{i}$ from this distribution, and then sample each $y_{i}$ from $N\left(\theta_{i}, 1\right)$. The selection region is chosen to be $S=\{y:|y|>2\}$ and we operate under the joint selection mechanism. That is, $\left(\theta_{i}, y_{i}\right)$ are sampled jointly and a confidence set is constructed for $\theta_{i}$ only if $\left|y_{i}\right|>2$.

Figure 1 shows the resulting optimal spending function for this particular combination of prior, sampling model, selection region, and selection mechanism. This spending function defines the family of selective hypothesis tests to create the Bayes-optimal confidence sets. For comparison, we also calculated the UMAU confidence sets from Fithian et al. (2014) which correspond to the flat spending function $w_{\text {saUMAU }}(\theta)=1 / 2$.

Figure 2 shows the sizes of each procedure's confidence sets $C(y)$ as a function of $y$. For reference, we have also plotted the marginal distribution of $y$ for the selected signals. Notably, 
the saFAB procedure gives a smaller confidence set for values of $y$ that are most frequently observed: for approximately $90 \%$ of the observations in this example, saFAB returns a smaller confidence set. For values of $y$ larger than approximately 3.6, saFAB give a wider confidence set than the intervals from Fithian et al. (2014), but observing a $y_{i}$ this large is relatively rare. As a result, the saFAB intervals are $12 \%$ shorter on average than the UMAU intervals.

Finally, Figure 3 shows the coverage of our saFAB method in comparison to two alternative methods: the non-selection adjusted confidence sets ("non-sa UMAU") and selection-adjusted Bayesian credible intervals ("saBayes"). Following Yekutieli (2012), the selection-adjusted posterior distribution is identical to the ordinary posterior distribution because of the mechanism of joint selection. Since the $\theta_{i}$ are drawn from the mixture in Eq. (9), we consider the two cases of $\theta=0$ and $\theta \neq 0$. The unadjusted confidence intervals have poor coverage, with nearly zero coverage for $\theta$ values equal to and near 0 , owing to selection bias. On the other hand, the selection-adjusted Bayesian credible intervals have correct coverage on average across all signals, but this coverage is highly non-uniform in $\theta$. In particular, since the credible interval nearly always includes 0 due to the presence of the point mass in the posterior, the coverage for $\theta=0$ is nearly 1 , while coverage is much lower for all other values of $\theta$. In the supplemental material, we show how this problem of poor coverage of credible intervals for nonzero $\theta$ is exacerbated when even greater prior mass is assigned for $\theta=0$. Our saFAB procedure, on the other hand, provides nominal coverage uniformly across the entire parameter space. Although not shown, the usual selection-adjusted UMAU confidence sets of Fithian et al. (2014) also exhibit the same uniform coverage; they are simply wider on average.

\section{Nonparametric empirical Bayes procedure}

\subsection{Estimating the prior}

In the previous section we introduced the saFAB procedure for situations where the prior $\pi(\theta)$ had a known parametric form. In cases where $\pi(\theta)$ is not known, however, it may not be desirable to specify a parametric form or elicit a subjective choice. For this situation, we propose a method for post-selection inference where a prior need not be fully specified but rather is estimated from the data using a nonparametric empirical Bayes approach. Throughout, we still assume that both the 



Figure 2: Comparison of sizes of selection-adjusted UMAU confidence sets (saUMAU) and our selection-adjusted FAB confidence sets as a function of observation size. For most observations (around $90 \%$ of the marginal density), saFAB returns a smaller confidence set, resulting in confidence sets that are $12 \%$ smaller on average.
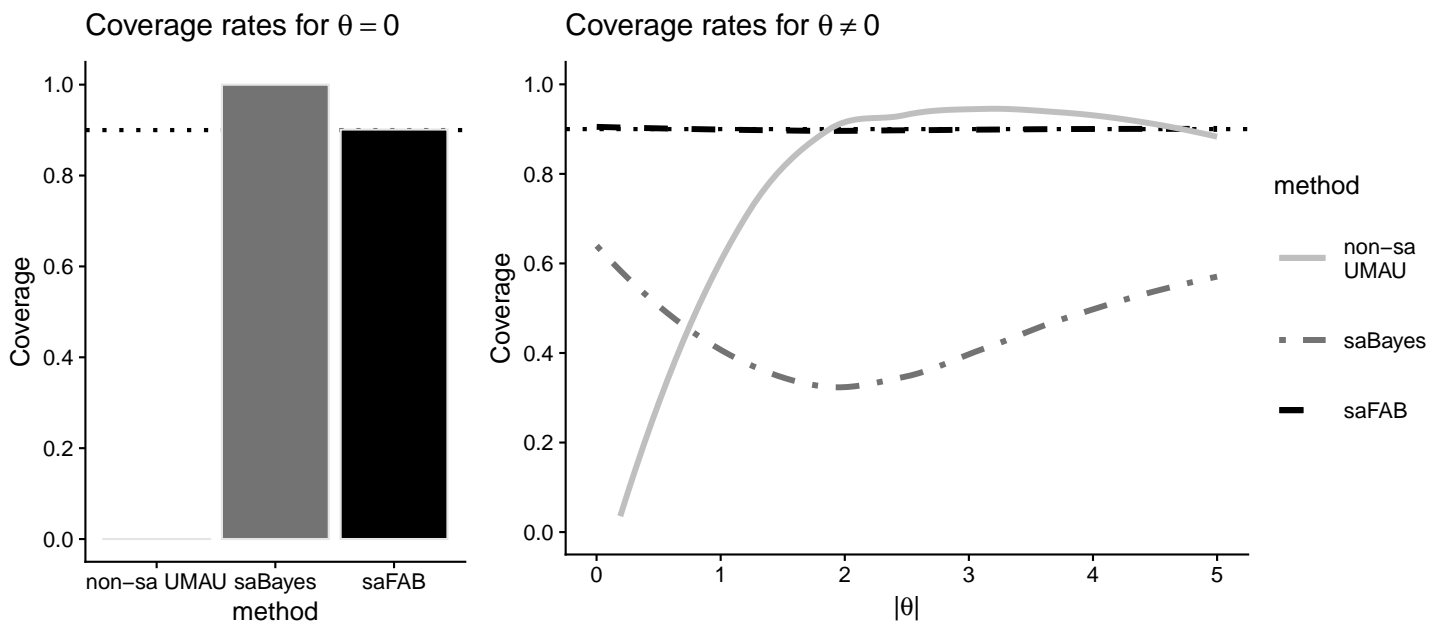

Figure 3: Results for toy example, comparing coverage rates for non-selection adjusted confidence sets (non-sa UMAU), selection-adjusted Bayesian posterior credible intervals (saBayes), and our selection-adjusted FAB confidence sets ( $\mathrm{saFAB}$ ). We delineate the two cases of $\theta=0$ (left) and $\theta \neq 0$ (right). Non-selection adjusted confidence sets have poor coverage throughout and selection-adjusted credible intervals only have correct coverage on average across the prior. saFAB confidence sets have uniform nominal coverage. 
sampling model $f(y ; \theta)$ and the selection mechanism are known, and that the selection rule $S$ has also been pre-specified. Our main theoretical result establishes that, under mild regularity conditions and with selection occurring jointly on $(\theta, y)$, our nonparametric method gives a consistent estimate of the true optimal spending function. In addition, our empirical results show that this data-driven strategy for constructing a spending function offers substantial efficiency improvements over the UMAU intervals, while losing very little compared to a hypothetical oracle-Bayes analysis in which the prior is known.

Recall that the optimal spending function $w^{\star}(\theta)$ is calculated by minimizing the objective function $H(w ; \theta)$ in Eq. (4), which involves the marginal density of selected observations $m_{S}(y)$. This marginal density in turn depends on both the selection mechanism, which is known, as well as the prior, which is unknown. The intuition of our approach is that if we are able to specify a prior whose corresponding marginal distribution matches the data well, we should also be able to recover a good estimate of the optimal spending function. Our nonparametric empirical Bayes approach exploits this fact by using a plug-in estimate $\hat{\pi}(\theta)$ for the prior distribution.

One challenge that arises in constructing such an estimate $\hat{\pi}(\theta)$ is that it must adapt to the data, yet still ensure valid post-selection sets. As implied by Proposition 7 from Yu and Hoff (2018), in order to retain nominal coverage of the procedure, the spending function-and therefore the estimate of the prior-cannot depend on the same data that we use to construct confidence sets. Otherwise, there is the potential for selection bias to be re-introduced via the mechanism of an overly optimistic prior. As an example, consider the data-dependent prior that concentrates all its probability mass on the observed value of $y: \pi\left(\theta_{i}\right)=\delta_{y_{i}}$. It is easy to show that, if we use our procedure to construct the optimal spending function under such an aggressively data-dependent prior, the resulting confidence sets will not retain nominal coverage.

To avoid a more subtle version of this pitfall, in practice we use a $K$-fold data-splitting approach, whereby the data are split into $K$ non-overlapping subsets $y_{1}, \ldots, y_{K}$. To construct confidence sets for the data points in fold 1, we first use the data in folds 2 through $K$ to form an estimate of the prior $\hat{\pi}(\theta)$. This gives us an estimate of the optimal spending function $\hat{w}(\theta)$, which we use to construct Bayes-optimal saFAB confidence sets for the data points in fold 1 . Note that the data in the other folds are used only to estimate the spending function, but the actual confidence interval for a single $\theta_{i}$ is constructed using this spending function and only the 
single observation $y_{i}$. We then repeat this process for each fold, always holding out the data from that fold when we estimate the prior. This ensures that the independence condition from Proposition 7 of Yu and Hoff (2018) is met. To actually form the estimate $\hat{\pi}(\theta)$, we use the method of predictive recursion from Newton (2002). We provide details of this approach in Algorithm 1 of Appendix A.

\subsection{Constructing the spending function}

We now assume that $\hat{\pi}(\theta)$ is given, and we use it to obtain an estimate of the optimal spending function $\hat{w}^{\star}(\theta)$ as follows. First, from the estimated prior $\hat{\pi}(\theta)$, we derive the corresponding marginal distribution of the data under selection, $\hat{m}_{S}(y)$, which is calculated by substituting $\hat{\pi}(\theta)$ in for $\pi(\theta)$ in either Eq. (6) or Eq. (8), depending on the selection mechanism. That is, the estimated selection-adjusted marginal is either

$$
\hat{m}_{S}^{(J)}(y)=\int \mathbf{1}(y \in S) \hat{\pi}(\theta) f(y ; \theta) / \hat{\operatorname{Pr}}(S) \mathrm{d} \theta=\hat{m}(y) \cdot \mathbf{1}(y \in S) / \hat{\operatorname{Pr}}(S) .
$$

for joint selection, where $\hat{\operatorname{Pr}}(S)=\iint_{S} f(y ; \theta) \hat{\pi}(\theta) \mathrm{d} y \mathrm{~d} \theta$ and $\hat{m}(y)=\int f(y ; \theta) \hat{\pi}(\theta) \mathrm{d} \theta$, or

$$
\hat{m}_{S}^{(C)}(y)=\mathbf{1}(y \in S) \int \hat{\pi}(\theta) f(y \mid \theta) / \operatorname{Pr}(S \mid \theta) \mathrm{d} \theta
$$

for conditional selection. This then defines the surrogate objective function

$$
\hat{H}(w ; \theta)=\hat{M}_{S}\left[F_{S}^{-1}(\alpha w+1-\alpha ; \theta)\right]-\hat{M}_{S}\left[F_{S}^{-1}(\alpha w ; \theta)\right]
$$

which serves as a proxy for the true (unknown) objective function $H(\theta ; w)$ in Eq. (4). Minimization of this objective function in turn gives the estimated optimal spending function $\hat{w}^{\star}(\theta)=$ $\arg \min _{w \in[0,1]} \hat{H}(w ; \theta)$. We then use this estimate of the optimal spending function to calculate confidence sets, in the manner described in $\$ 2, \mathrm{E}$

\subsection{Main consistency result}

The key statistical question that arises from our procedure is whether the minimizer of the surrogate objective $\hat{H}(w ; \theta)$ is a good estimate for the minimizer of the true, unknown objective 
$H(w ; \theta)$. We now turn to our main theoretical result, which establishes that, under mild conditions, the answer is yes, at least in the case of joint selection. Our proof builds upon the foundation laid by Tokdar et al. (2009), who give conditions under which predictive recursion will yield an estimate $\hat{m}(y)$ that converges to the true marginal density $m(y)$. From their result, it is easy to demonstrate that the estimated selection-adjusted marginal density for joint selection, $\hat{m}_{S}^{(J)}(y)$ in Eq. (10) also converges to the true density $m_{S}^{(J)}$ in Eq. (6). Our theorem hinges on using this fact to show that the worst-case distance from any minimizer $\hat{w}^{\star}(\theta)$ of the surrogate objective function $\hat{H}(\cdot ; \theta)$ to the set of minimizers of $H(; \theta)$ converges to zero in probability; see Eq. (13) below. Thus, for any minimizer $\hat{w}^{\star}(\theta)$ of $\hat{H}(\cdot ; \theta)$ there exists a minimizer $w^{\star}(\theta)$ of $H(\cdot ; \theta)$ which is close to $\hat{w}^{\star}(\theta)$. Furthermore, we show that the value of $H(\cdot ; \theta)$ at any minimizer of $\hat{H}(\cdot ; \theta)$ converges to the optimal value of $H(\cdot ; \theta)$. These results require the same conditions in Tokdar et al. (2009) that are necessary to ensure the Kullback-Leibler convergence of the predictiverecursion estimator. The explicit assumptions are given in Appendix B. The first condition has to do with the choice of the weights in the predictive recursion algorithm, whereas the remaining are regularity assumptions on the model. These include an identifiability condition of the mixing density when the likelihood is $f(\cdot ; \theta)$; the requirement that $f(y ; \theta)$ is bounded and continuous as a function of $\theta$; and two boundedness properties involving the behavior of $f$. As shown in Tokdar et al. (2009) these conditions all hold for the Gaussian model.

Theorem 1 Suppose that the selection region is $S=\{y:|y|>t\}$ for some $t>0$ with selection occurring jointly on $(\theta, y)$, and that $f(\cdot ; \theta)$ has as support the real line. Also suppose that the estimated prior $\hat{\pi}(\theta)$ is calculated using predictive recursion as outlined in Algorithm 1 using a subset of the data that grows with $n$. If Assumptions A1-A5 from Tokdar et al. (2009) are met by $f$ (see Appendix B), then for a fixed $\theta$ the following statements hold:

- Let $\Omega_{0}$ be the set of minimizers of $H(\cdot ; \theta)$, and $\hat{\Omega}$ the set of minimizers of $\hat{H}(\cdot ; \theta)$. Assume that $\Omega_{0} \neq \emptyset$. If $q:=F_{S}(t, \theta) \leq \alpha$ and $q / \alpha \notin \Omega_{0}$ then we require that $\lim _{w \rightarrow(q / \alpha)^{+}} H(w ; \theta)>$ $\min _{\tilde{w} \in[0,1]} H(\tilde{w} ; \theta)$. Then for every $\epsilon>0$ we have

$$
\lim _{n \rightarrow \infty} \operatorname{Pr}\left(\sup _{\hat{w}^{\star} \in \hat{\Omega}} \inf _{w^{\star} \in \Omega_{0}}\left|\hat{w}^{\star}-w^{\star}\right| \geq \epsilon\right)=0 .
$$

Thus, the worst-case distance from $\hat{\Omega}$ to $\Omega_{0}$ converges to zero in probability. 
- Almost surely, if $\hat{w}^{\star} \in \hat{\Omega}$ then

$$
\lim _{n \rightarrow \infty} H\left(\hat{w}^{\star} ; \theta\right)=H\left(w^{\star} ; \theta\right),
$$

for some $w^{\star} \in \Omega_{0}$.

Proof 1 See Appendix $C$

We notice that when $q=F_{S}(t ; \theta) \leq \alpha$ and $q / \alpha \notin \Omega_{0}$ the conclusion (13) requires an extra condition. If such condition is violated, thus,

$$
\lim _{w \rightarrow(q / \alpha)^{+}} H(w ; \theta)=\min _{w \in[0,1]} H(w ; \theta)
$$

then 13 will hold replacing $\Omega_{0}$ with $\Omega_{0} \cup\{q / \alpha\}$. This is reasonable since when 15 holds even minimization of $H$ over a finite grid of points in $[0,1]$ could lead to points that are arbitrarily close to $q / \alpha$ as the grid size grows.

An important implication of Theorem 1 has to do with constructed confidence sets. In particular, let $\theta_{1}, \ldots, \theta_{N}$ fixed points in the real line. If all the assumptions in Theorem 1 hold for each $\theta_{j}$ and $F_{S}\left(t ; \theta_{j}\right) / \alpha$ is not a minimizer of $H\left(\cdot ; \theta_{j}\right)$ for all $j=1, \ldots, N$, then for each $j$ there exists a $w^{\star}\left(\theta_{j}\right)$ minimizer of $H\left(\cdot ; \theta_{j}\right)$ such that for all $\epsilon>0$,

$$
\operatorname{pr}\left[\max _{j=1, \ldots, N} \nu\left\{\left(A_{\hat{w}^{\star}\left(\theta_{j}\right)}^{S} \backslash A_{w^{\star}\left(\theta_{j}\right)}^{S}\right) \cup\left(A_{w^{\star}\left(\theta_{j}\right)}^{S} \backslash A_{\hat{w}^{\star}\left(\theta_{j}\right)}^{S}\right)\right\} \geq \epsilon\right] \rightarrow 0,
$$

where $\nu$ is the Lebesgue measure. Thus, the acceptance regions for the grid points based on the estimated spending function will be closed to those based on the true spending function. Consequently, if a new data point is collected then the confidence set based on the estimated spending function and the grid of points will be equals to that based on the same grid and the true spending function, with probability approaching one (see Corollary 1 in Appendix D. If instead (15) holds for some $\theta_{j}$ or $F_{S}\left(t ; \theta_{j}\right) / \alpha$ is a minimizer of $H\left(\cdot ; \theta_{j}\right)$, then such $\theta_{j}$ would have to be excluded from the statement in (16) and from that of Corollary 1 . This is due to the discontinuity of $F_{S}^{-1}\left(; \theta_{j}\right)$ at $F_{S}\left(t ; \theta_{j}\right)$.

On another note, we restrict ourselves to selective inference with a selection region of the form $S=\{y:|y|>t\}$ for $t>0$. However, because this result mostly hinges on convergence 
of $\hat{m}$ to $m$, it also holds for asymmetric and one-sided selection regions. In fact, for one sided regions the extra regularity condition on $q$ is not needed for Theorem 1 . Similarly, in such setting (16) and Corollary 1 hold without conditions on $q$. Theorem 1 also applies when constructing non-selective confidence sets, including the situation studied in Yu and Hoff (2018). A similar result is more difficult to show for conditional selection, as the selection-adjusted marginal in this case has the much less tractable form given in Eq. (11).

We also note that the procedure comes in with a built-in form of robustness. Even if the prior is not well estimated for a given sample, by construction the procedure will still give confidence sets that retain nominal coverage, as long as the spending function is not data-dependent. In other words, prior misspecification can mitigate the efficiency of confidence sets, but not their coverage.

\section{Results on simulated and real data}

\subsection{Case of well-specified prior}

We now investigate the performance of our saFAB procedure to the usual selective confidence set, i.e. the UMAU confidence sets developed by Reid et al. (2014), at the $\alpha=0.1$ level. Specifically, we will check that nominal coverage is maintained, and also compare the efficiency (size) of the confidence sets. We consider saFAB confidence sets from three variants of constructing the spending function: (i) the "oracle" case where we know the parametric form and the hyperparameters of the prior, (ii) the "parametric empirical Bayes" (PEB) case where we assume a parametric form of the prior, and estimate hyperparameters via maximum marginal likelihood estimation using five-fold data-splitting, and (iii) the "nonparametric empirical Bayes" (NPEB) case as described in the $\$ 3$, where estimate the prior $\pi(\theta)$ from the data via the predictive recursion algorithm of Newton (2002), using five-fold data-splitting.

We sample $\theta_{i}$ from several considered distributions, and generate data $y_{i}$ from the Gaussian distribution centered on $\theta_{i}$ with unit variance. The sampling variance is assumed known for each method of constructing confidence sets.

First we consider signals generated from the point mass-Gaussian mixture in Eq. (9) for the toy example in $\$ 2.3$ with the hyperparameters set to $p=0.2$ and $\tau^{2}=3$. We simulate 2,000,000 


\begin{tabular}{llll}
\hline Method & Coverage & Average size & Relative average size \\
\hline Oracle & $0.8990(0.0095)$ & $3.3493(0.0014)$ & $0.8956(0.0004)$ \\
PEB & $0.8990(0.0095)$ & $3.3510(0.0014)$ & $0.8960(0.0004)$ \\
NPEB & $0.8995(0.0095)$ & $3.3555(0.0014)$ & $0.8972(0.0004)$ \\
UMAU & $0.8993(0.0095)$ & $3.7399(0.0016)$ & $1.0000(0.0004)$ \\
\hline
\end{tabular}

Table 1: Comparison of performance for the well specified prior case under joint selection. We consider saFAB when the prior is assumed known (oracle), saFAB when the prior form is known and hyperparameters are estimated from the data (parametric empirical Bayes, PEB), saFAB when no prior form is assumed but is rather estimated from the data using predictive recusion (NPEB), and the existing selective confidence sets resulting from inverting unbiased tests (UMAU).

\begin{tabular}{llll}
\hline Method & Coverage & Average size & Relative average size \\
\hline Oracle & $0.9005(0.0095)$ & $3.5138(0.0021)$ & $0.9385(0.0006)$ \\
PEB & $0.9004(0.0095)$ & $3.5113(0.0021)$ & $0.9378(0.0006)$ \\
NPEB & $0.8999(0.0095)$ & $3.5123(0.0021)$ & $0.9381(0.0005)$ \\
UMAU & $0.8998(0.0095)$ & $3.7441(0.0016)$ & $1.0000(0.0004)$ \\
\hline
\end{tabular}

Table 2: Comparison of performance for the well specified prior case under conditional selection

total signals, structured in 1000 batches as follows. For each batch we make $n=2000$ draws from the mixture model for $\theta_{i}$ and generate $y_{i}$ from $N\left(\theta_{i}, 1\right)$. For conditional selection, these $\theta_{i}$ are generated once and used for each batch, while for the joint selection case they are generated anew each time. The selection rule for determining the $\theta_{i}$ for which to construct confidence sets is $S=\{y:|y|>2\}$. For these selected $\theta_{i}$ we construct $90 \%$ confidence sets under the four considered methods, and then for each method we calculate the proportion of confidence sets which cover the true $\theta_{i}$, as well as the average size of the confidence sets.

Tables 1 and 2 show the results for joint and conditional selection, respectively. The coverage of each method hems closely to the nominal rate. The oracle saFAB procedure, however, gives confidence sets that are on average approximately 10\% (joint selection) and $6 \%$ (conditional selection) more efficient than the existing selective confidence set procedure (UMAU). The efficiency of the parametric empirical Bayes saFAB procedure closely hems to that of the oracle. Notably, the nonparametric empirical Bayes variant of saFAB also comes very close to the oracle, even though it makes no assumption of the form of the prior. 


\subsection{Case of misspecified prior}

Now we compare the performance of the nonparametric procedure against the parametric procedure under a misspecified prior. That is, for the parametric procedure we assume the same parametric form of a point mass-Gaussian mixture for the prior, but in reality the $\theta_{i}$ are drawn from a different distribution. We study two different scenarios of misspecification: one where the non-zero $\theta_{i}$ come from a bimodal distribution with both modes separated from zero, that is

$$
\theta_{i} \sim(p / 2) \mathcal{N}\left(-\mu, \tau^{2}\right)+(p / 2) \mathcal{N}\left(\mu, \tau^{2}\right)+(1-p) \delta_{0}
$$

with $\tau^{2}=1 / 4$ and $p=0.2$, and one where they come from a skewed, unimodal nonzero-centered distribution, that is

$$
\theta_{i} \sim p\{\mu+\text { Exponential }(\lambda)\}+(1-p) \delta_{0}
$$

with $\mu=1$ and $\lambda=1$.

In both cases, we simulate 1000 batches of $n=2000$ pairs of $\left(\theta_{i}, y_{i}\right)$, and we retain the same

selection region, $S=\{y:|y|>t\}$. For brevity, we only consider selection acting jointly on $\left(\theta_{i}, y_{i}\right)$.

Tables 3 and 4 show the results. Again, each method maintains nominal coverage. That this is true for the parametric saFAB procedure shows that even under prior misspecification, saFAB gives correct coverage. For both considered priors, the oracle saFAB gives confidence sets which are about 11-12\% more efficient than the UMAU procedure. Interestingly, the parametric and nonparametric saFAB procedures show only minor differences in their efficiency gains, and come close to the oracle. Whereas nonparametric slightly outperforms parametric in the case of the skewed alternative density, this result is reversed for the bimodal alternative density. This latter fact is surprising given that the assumed prior in the parametric procedure is incorrect, and is likely explained by a high estimated variance for the unimodal alternative density. 


\begin{tabular}{llll}
\hline Method & Coverage & Average size & Relative average size \\
\hline Oracle & $0.9012(0.0094)$ & $3.3941(0.0007)$ & $0.8884(0.0002)$ \\
PEB & $0.9038(0.0093)$ & $3.4148(0.0007)$ & $0.8938(0.0002)$ \\
NPEB & $0.9017(0.0094)$ & $3.4335(0.0008)$ & $0.8987(0.0002)$ \\
UMAU & $0.8999(0.0095)$ & $3.8204(0.0009)$ & $1.0000(0.0002)$ \\
\hline
\end{tabular}

Table 3: Comparison of performance when the prior is misspecified for the parametric empirical Bayes saFAB procedure. The true density for $\theta$ is a mixture of a point mass at zero and a bimodal alternative density, and signals are selected under joint selection.

\begin{tabular}{llll}
\hline Method & Coverage & Average size & Relative average size \\
\hline Oracle & $0.8940(0.0097)$ & $3.3149(0.0011)$ & $0.8808(0.0003)$ \\
PEB & $0.8937(0.0097)$ & $3.3941(0.0011)$ & $0.9019(0.0003)$ \\
NPEB & $0.9070(0.0092)$ & $3.3672(0.0019)$ & $0.8947(0.0005)$ \\
UMAU & $0.9002(0.0095)$ & $3.7635(0.0014)$ & $1.0000(0.0004)$ \\
\hline
\end{tabular}

Table 4: Comparison of performance when the prior is misspecified for the parametric empirical Bayes saFAB procedure. The true density for $\theta$ is a mixture of a point mass at zero and a skewed alternative density, and signals are selected under joint selection.

\subsection{Data-dependent thresholding}

So far we have so far presumed that the selection set $S$ is specified $a$ priori, so that the selectionadjusted sampling model is well-defined. Now we investigate the use of data-adaptive selection sets, where the selection set $S$ is now a random variable and so the truncated sampling model in Eq. (1) in not well-defined. We focus on the popular Benjamini-Hochberg (BH) procedure (Benjamini and Hochberg, 1995) for controlling the false discovery rate (FDR) to decide the selection rule. To briefly summarize the $\mathrm{BH}$ procedure, suppose we have $m$ null hypotheses $H_{01}, \ldots, H_{0 m}$ all with corresponding $p$-values $p_{1}, \ldots, p_{m}$, and let $p_{(k)}$ represent the $k$ th smallest $p$-value with corresponding null hypothesis $H_{(k)}$. Rejecting all null hypotheses $H_{(1)}, \ldots, H_{\left(k^{\star}\right)}$ with

$$
k^{\star}=\max \left\{k: p_{(k)} / k \leq q^{\star} / m\right\}
$$

bounds the FDR at $q^{\star}$, where the FDR is the expected proportion of rejected null hypotheses which are actually true. This is a data-dependent rule for selection because we must calculate the $p$-values from the data to decide the selection rule. Reid et al. (2014) characterize the $\mathrm{BH}$ 


\begin{tabular}{lccc}
\hline Method & Average coverage & Average size & Relative average size \\
\hline Oracle & $0.9028(0.0094)$ & $3.7088(0.0019)$ & $0.9158(0.0005)$ \\
PEB & $0.9033(0.0093)$ & $3.7072(0.0019)$ & $0.9154(0.0005)$ \\
NPEB & $0.9032(0.0093)$ & $3.7084(0.0022)$ & $0.9157(0.0005)$ \\
UMAU & $0.9088(0.0091)$ & $4.0500(0.0014)$ & $1.0000(0.0003)$ \\
\hline
\end{tabular}

Table 5: Comparison of performance when using a data-dependent threshold, via the BenjaminiHochberg procedure, for the selection set, and signals are selected under joint selection.

procedure as an affine linear constraint for use in the selection-adjusted likelihood for constructing UMAU confidence intervals, but here we are interested in investigating the performance of confidence sets constructed by simply treating the $\mathrm{BH}$ procedure as a simple data-dependent thresholding problem.

We perform a simulation study to see if nominal coverage is still upheld using this approach. We generate data in an identical way as done in $\$ 4.1$. We only consider the case of joint selection. Each two-sided $p$-value for the point null hypothesis $H_{0 i}: \theta_{i}=0$ is then given by $p_{i}=2 \Phi\left(-\left|y_{i}\right|\right)$, where $\Phi(\cdot)$ is the standard normal cumulative density function. We use the BH rule in Eq. (17) to choose which $\theta_{i}$ to be considered for inference. The selection set for constructing the selectionadjusted likelihood is now considered to be $S=\left\{y:|y|>\left|y_{\left(k^{\star}+1\right)}\right|\right\}$, i.e. the threshold is determined by the largest $y_{i}$ in magnitude of those not chosen by the $\mathrm{BH}$ procedure. We use $q^{\star}=0.2$ as the target FDR for the decision rule, and again construct $90 \%$ confidence sets for each method, performing 1000 simulations.

The results are presented in Table 5. We appear to recover nominal coverage for each method. This suggests that using the $\mathrm{BH}$ procedure as a data-dependent selection rule will still give valid confidence sets for the saFAB procedure.

\subsection{Analysis of neural synchrony data}

Finally, we apply the nonparametric saFAB procedure to a real dataset to show how, with minimal assumptions about the prior, we may still construct confidence sets which have significant gains in efficiency over the UMAU confidence sets. To do so, we analyze the neural synchrony data published in Smith and Kohn (2008) and Kelly et al. (2010), and re-analyzed by Scott et al. (2015). The goal of this application is to identify fine-time-scale neural interactions ("synchrony") among 


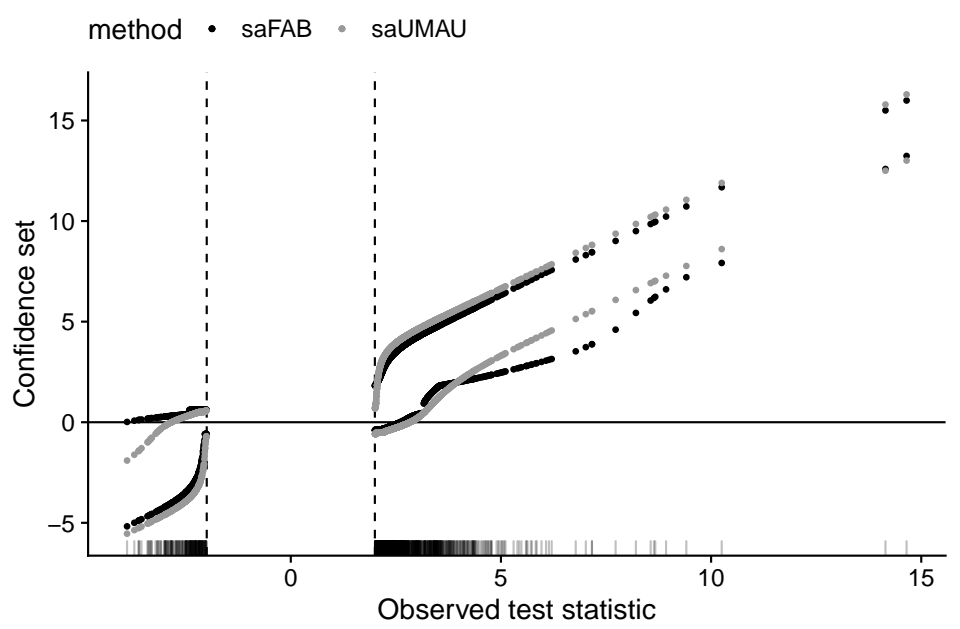

Figure 4: Comparison of confidence intervals from saFAB and UMAU. Our saFAB procedure gives confidence intervals that are $11.3 \%$ shorter on average, with $85 \%$ of constructed intervals being shorter.

many neurons recorded simultaneously by a multi-electrode array. The experiment from which the data are drawn produced thousands of pairwise test statistics, each representing the magnitude of interaction between a single pair of neurons.

Kelly et al. (2010) provide full details of the data and the experiment. For our purposes, the relevant fact is that the data for each neuron pair can be assumed to take the form $z_{i} \sim$ $N\left(\theta_{i}, 1\right)$ (we use $i$ to index pairs, which can be thought of as edges in a network). Here $\theta_{i}$ can be interpreted as a $\log$ rate ratio: that is, $e^{\theta_{i}}$ represents how much more often, in multiplicative terms, the two neurons in pair $i$ fire together, compared to the rate one would expect if they were firing independently. Thus if $\theta_{i} \approx 0$, the two neurons are plausibly independent, while if $\theta_{i}$ is substantially larger than zero, they exhibit an interesting pattern of fine-time-scale synchronous firing. The case $\theta_{i}<0$ is less well understood scientifically, but potentially interesting as well.

In our analysis, we assume no parametric form of the prior for $\theta$, preferring instead to use the nonparametric saFAB procedure outlined in $\$ 3$ to estimate the prior via predictive recursion. We use $S=\{y:|y|>2\}$ as a very liberal selection region. Here selection acts jointly on $\theta_{i}$ and $y_{i}$, since we will form confidence sets only for those $\theta_{i}$ that meeting an initial screen of significance. Figure 4 shows the saFAB confidence sets as compared to the selection-adjusted UMAU confidence sets. The asymmetry in these intervals reflects that fact that most signals in the data set corresponding to presumed cases where $\theta_{i}>0$ (synchrony enhancement), rather than 
$\theta_{i}<0$ (synchrony suppression). Indeed, there are clear scientific reasons to suspect than many neuron pairs will have $\theta_{i}>0$, but the case $\theta_{i}<0$ would be unusual. Our analysis detects this fact without having to assume it, and adapts to it via the choice of the spending function.

For the large majority selected test statistics, about $85 \%$, our saFAB method produces a shorter interval than the UMAU method. The saFAB intervals are considerably more efficient; the average width of the saFAB intervals is 3.38 , about $11.3 \%$ smaller than the average width of UMAU intervals, which is 3.81. While the saFAB intervals are wider for some of the larger test statistics, these observations are relatively few. Indeed, because the marginal distribution of selected test statistics is tightly concentrated near the boundary of the selection region, these are the intervals which receive the efficiency gains of our procedure by design. Having tighter intervals for these observations is important since there is a greater chance of them being false positives.

We emphasize that our analysis complements, rather than competes with, the analyses in Kelly et al. (2010) and Scott et al. (2015). In those papers, the goal was to discover interesting pairs of neurons, i.e. to test which pairs have $\theta_{i} \neq 0$. Our analysis takes such a test as a starting point. Using the techniques developed here, we are able conduct valid frequentist inference for

the discovered $\theta_{i}$ 's, while exploiting their probabilistic structure via a prior, and simultaneously controlling for post-selection inference. Because $\theta_{i}$ has a useful neurophysiological interpretation as a log relative rate, quantifying uncertainty about its magnitude can in this manner can add substantially to the analyses conducted by previous authors.

\section{Discussion}

The central argument of this paper has been that the use of a prior can play a decisive and favorable role in constructing optimal selection-adjusted confidence sets. Here we identify three open questions. First, an interesting future line of work would be to undertake a formal investigation of the performance of the saFAB method under data-dependent thresholding, such as the Benjamini-Hochberg procedure. Our simulation-based results suggest that the performance is excellent, while theoretical investigations of the UMAU method show that coverage does indeed hold because the selection rule satisfies a key polyhedral condition (e.g. Lee et al., 2016). But more theoretical work is necessary to merge these lines of reasoning. Second, it may be of interest to create intervals which have minimum expected size with respect to the marginal distribution of 
selected signals (i.e. $\pi_{S}(\theta)$ in the notation of Yekutieli, 2012) rather than with respect to the entire distribution of signals, i.e. $\pi(\theta)$. We leave avenue this to future work. Finally, there is much research left to be done in this direction of merging Bayesian and frequentist thinking in other areas of inference where selection takes place. Current ongoing work extends our methods to regression, spatial hotspot detection, and subgroup identification in causal inference. Preliminary results show promising improvements in these areas.

\section{A Predictive recursion}

For the nonparametric saFAB procedure, we assume that the data arise from the model

$$
y_{i} \sim f\left(y_{i} ; \theta_{i}\right), \quad \theta_{i} \sim \pi(\theta)
$$

where the sampling density $f(y ; \theta)$ is known, and the prior $\pi(\theta)$ is to be estimated. To do so, we use predictive recursion method of Newton (2002) to estimate the mixing density $\pi(\theta)$ from the observations $y_{1}, \ldots, y_{n}$.

We begin with an intial guess $\pi^{[0]}$ and a sequence of weights $\gamma^{[i]} \in(0,1)$. For $i=1, \ldots, n$, we recursively compute the update

$$
\begin{aligned}
m^{[i-1]}\left(y_{i}\right) & =\int_{\mathbb{R}} f\left(y_{i} ; u\right) \pi(\mathrm{d} u) \\
\pi^{[i]}(\mathrm{d} u) & =\left(1-\gamma^{[i]}\right) \pi^{[i-1]}(\mathrm{d} u)+\gamma^{[i]} \frac{f\left(y_{i} ; \theta\right) \pi^{[i-1]}(\mathrm{d} u)}{m^{[i-1]}\left(y_{i}\right)} .
\end{aligned}
$$

Algorithm 1 details our implementation. We sweep through the data 10 times, each time randomizing the sweep order over the data. In practice, the prior $\pi(\theta)$ is computed on a grid, and the integral in Eq. (18) is computed using the trapezoid rule. Tokdar et al. (2009) give conditions on the weights $\gamma^{[i]}$ to lead to almost-sure weak convergence of the PR estimate to the true mixing distribution. In the case that the mixture model is misspecified, they show that the PR estimate converges in total variation to the mixing density that minimizes the Kullback-Leibler divergence to the truth. Specifically, the conditions for convergence are satisfied by $\gamma^{[i]}=(i+1)^{-a}$, and we use the default value $a=0.67$ recommended by Tokdar et al. (2009). 
Input : Data $y_{1}, \ldots, y_{n}$; sampling model $y_{i} \sim f\left(y_{i} ; \theta_{i}\right)$; intial guess $\pi^{[0]}(\theta)$.

for $i=1, \ldots, n$ do

$$
\begin{aligned}
g^{[i]}(\theta) & =f\left(y_{i} ; \theta\right) \pi_{1}^{[i-1]}(\theta) \quad \text { (discrete grid) } \\
m^{[i]} & =\int_{\mathbb{R}} g^{[i]}(\theta) \mathrm{d} \theta \quad(\text { trapezoid rule) } \\
\pi^{[i]}(\theta) & =\left(1-\gamma^{[i]}\right) \cdot \pi_{1}^{[i-1]}(\theta)+\gamma^{[i]} \cdot\left(\frac{f\left(y_{i} ; \theta\right) \pi^{[i-1]}(\theta)}{m^{[i]}}\right)
\end{aligned}
$$

Output: Estimate $\hat{\pi}(\theta)=\pi^{[n]}(\theta)$.

Algorithm 1: Predictive recursion algorithm for estimating the prior, which is then used for estimating the optimal spending function as outlined in $\$ 3$.

\section{B Assumptions for main result}

Here we state the assumptions from Tokdar et al. (2009) that are required for Theorem 1 .

A1. The weights $\left\{\gamma^{[i]}\right\}_{i=1}^{\infty}$ in Algorithm 1 are chosen to satisfy $\sum_{i=1}^{\infty} \gamma^{[i]}=\infty$ and $\sum_{i=1}^{\infty}\left(\gamma^{[i]}\right)^{2}<$ $\infty$.

A2. The map $F \rightarrow \int f(y ; \theta) F(d \theta)$ is injective.

A3. For each $y$, the map $\theta \rightarrow f(y ; \theta)$ is bounded and continuous.

A4. For all $\epsilon>0$, and $\mathcal{X}_{0}$ compact set, there exists a compact set $\Theta_{0}$ such that $\int_{\mathcal{X}_{0}} f(y ; \theta) d y<\epsilon$ for all $\theta \notin \Theta_{0}$.

A5. There exists a constant $B<\infty$ such that for all $\theta_{1}, \theta_{2}$ and $\theta_{3}$ we have that

$$
\int\left\{\frac{f\left(y ; \theta_{1}\right)}{f\left(y ; \theta_{2}\right)}\right\}^{2} f\left(y ; \theta_{3}\right) d y<B
$$

\section{Proof of main result}

Throughout we drop the dependence on $\theta$ and simply refer to $H(\cdot, \theta)$ as $H$. To prove Theorem 1 . we first notice that

$$
\sup _{y \in R}\left|M_{S}(y)-\hat{M}_{S}(y)\right| \leq \int\left|m_{S}(y)-\hat{m}_{S}(y)\right| d y \rightarrow^{a . s} 0
$$


by Theorem 2 from Tokdar et al. (2009). Hence, $\hat{H} \rightarrow H$ uniformly, almost surely.

Next, we observe that the function $M_{S}$ is continuous everywhere as by Fubini's theorem

$M_{S}(y)=\int_{-\infty}^{y} m_{S}(u) \mathrm{d} u=\int_{-\infty}^{y} \int f_{S}(u ; \theta) \pi(\mathrm{d} \theta) \mathrm{d} u=\iint_{-\infty}^{y} f_{S}(u ; \theta) \mathrm{d} u \pi(\mathrm{d} \theta)=\int F_{S}(y ; \theta) \pi(\mathrm{d} \theta)$.

Furthermore, the function $F_{S}^{-1}$ is continuous everywhere except at $q=F_{S}(-t ; \theta)=F_{S}(t ; \theta)$, a point where $F_{S}^{-1}$ is left continuous. Therefore, $H$ is continuous everywhere except perhaps in a set $\Lambda$ containing at most two different points. Additionally, $H$ is left continuous everywhere.

With the above in mind, the proof of (13) uses Wald's argument, in the spirit of Theorem 5.14 from van der Vaart (1998), or the original paper Wald (1949). We start by taking $w_{0} \in(0,1)$ minimizer of $H$. We also set

$$
\mathcal{T}:=[0,1] \backslash\left(\Omega_{0} \cup \Lambda\right)
$$

where $\Lambda$ is the set of discontinuities of $H$. Then $\Lambda \subset\{(q+\alpha-1) / \alpha, q / \alpha\}$. If $w=(q+\alpha-1) / \alpha \in$ $[0,1)$, we observe that

$$
\begin{aligned}
\lim _{\tilde{w} \rightarrow w^{+}} H(\tilde{w}) & =M_{S}\left[\lim _{\tilde{q} \rightarrow q^{+}} F_{S}^{-1}(\tilde{q})\right]-M_{S}\left[F_{S}^{-1}(q+\alpha-1)\right] \\
& >M_{S}\left[F_{S}^{-1}(q)\right]-M_{S}\left[F_{S}^{-1}(q+\alpha-1)\right] \\
& =H(w) \\
& \geq \inf _{\tilde{w} \in[0,1]} H(\tilde{w}) .
\end{aligned}
$$

Therefore, by our assumption on $q$ (when $q \leq \alpha$ ), we have that

$$
\min _{w \in \Lambda \backslash \Omega_{0}} \lim _{\tilde{w} \rightarrow w^{+}} H(\tilde{w})-\inf _{w \in[0,1]} H(w)>0 .
$$

Hence, for $\epsilon>0$ is small enough it holds that

$$
\min _{w \in \Lambda \backslash \Omega_{0}} \lim _{\tilde{w} \rightarrow w^{+}} H(\tilde{w})-\inf _{w \in[0,1]} H(w)>2 \epsilon .
$$

Next, let $w \in \mathcal{T}$ be fixed, and let $U_{l}$ be a decreasing sequence of intervals around $w$, with 
diameter $\left(U_{l}\right)$ converging to zero. Let

$$
H(U)=\inf _{\tilde{w} \in U} H(\tilde{w})
$$

for $U \subset[0,1]$. Notice that $H\left(U_{l}\right) \geq H\left(w_{0}\right)$ for all $l$. Suppose that $\lim _{l \rightarrow \infty} H\left(U_{l}\right)=H\left(w_{0}\right)$. Then there exists a sequence $\left\{w_{l}\right\}$ with $w_{l} \in U_{l}$ such that $H\left(w_{l}\right) \rightarrow H\left(w_{0}\right)$. Hence, by the Bolzano-Weierstrass theorem, we have that for a subsequence of $\left\{w_{l}\right\}$, say $\left\{w_{l_{k}}\right\}$ it holds that $w_{l_{k}} \rightarrow w^{\prime}$ for some $w^{\prime} \in[0,1]$. However, since $U_{l_{k}}$ converges to $w$, it must be the case that $w^{\prime}=w$. Hence,

$$
H(w)=H\left(w^{\prime}\right)=\lim _{k \rightarrow \infty} H\left(w_{l_{k}}\right)=H\left(w_{0}\right),
$$

which contradicts $w \in \mathcal{T}$. Therefore, for all $w \in \mathcal{T}$ we have that $H\left(U_{w}\right)>H\left(w_{0}\right)$ for some small enough neighborhood $U_{w}$ of $w$.

If $\Lambda \backslash \Omega_{0} \neq \emptyset$, let $w \in \Lambda \backslash \Omega_{0}$ and let $U_{l}$ be a decreasing sequence of intervals around $w$, with diameter $\left(U_{l}\right)$ converging to zero. Suppose that $\lim _{l \rightarrow \infty} H\left(U_{l}\right)=H\left(w_{0}\right)$. Then there exists a sequence $\left\{w_{l}\right\}$ with $w_{l} \in U_{l}$ such that $H\left(w_{l}\right) \rightarrow H\left(w_{0}\right)$. Hence, by the Bolzano-Weierstrass theorem, we have that for a subsequence of $\left\{w_{l}\right\}$, say $\left\{w_{l_{k}}\right\}$ it holds that $w_{l_{k}} \rightarrow w$, and either $w_{l_{k}} \geq w$ for all $k$ or $w_{l_{k}} \leq w$ for all $k$. If $w_{l_{k}} \geq w$ for all $k$ then

$$
\lim _{\tilde{w} \rightarrow w^{+}} H(w)=\lim _{k \rightarrow \infty} H\left(w_{l_{k}}\right)=H\left(w_{0}\right)
$$

which contradicts (19). On the other hand if, $w_{l_{k}} \leq w$ for all $k$, then by the left continuity of $H$ we arrive at 20 which once again contradicts $(19)$. Therefore, for all $w \notin \Omega_{0}$ we have that $H\left(U_{w}\right)>H\left(w_{0}\right)$ for some small enough neighborhood $U_{w}$ of $w$.

Now, notice that the set $B$ is compact, where

$$
B:=\left\{w \in[0,1]: \quad \inf _{\tilde{w} \in \Omega_{0}}|w-\tilde{w}| \geq \epsilon\right\} .
$$

Clearly, $B$ can be covered by the intervals $\left\{U_{w}: w \in B\right\}$. Hence, there exists $U_{w^{(1)}}, \ldots, U_{w^{(p)}}$ that cover $B$. Therefore,

$$
\inf _{w \in B} \hat{H}(w) \geq \inf _{w \in \cup_{j=1}^{p} U_{w}^{(j)}} \hat{H}(w) \rightarrow \rightarrow_{\text {a.s }} \inf _{w \in \cup_{j=1}^{p} U_{w}^{(j)}} H(w)>H\left(w_{0}\right)
$$


where the limit follows from the, almost sure, uniform convergence of $\hat{H}$ to $H$. However, if $\hat{w}^{\star} \in B \cap \hat{\Omega}_{n}$, then

$$
\inf _{w \in B \cap \hat{\Omega}_{n}} \hat{H}(w)=\inf _{w \in B} \hat{H}(w)=\inf _{w \in[0,1]} \hat{H}(w) \leq \inf _{w \in[0,1]} H(w)+o_{P}(1) .
$$

Therefore,

$$
\left\{\hat{w}^{\star} \in B \cap \hat{\Omega}_{n}\right\} \subset\left\{\inf _{w \in B} \hat{H}(w) \leq H\left(w_{0}\right)+o_{P}(1)\right\},
$$

but, according to [21], the event on the right has probability that converges to zero as $n \rightarrow 0$. This proves (13). To prove (14), notice that

$0 \leq H\left(\hat{w}^{\star}\right)-H\left(w^{\star}\right) \leq \hat{H}\left(\hat{w}^{\star}\right)-\hat{H}\left(w^{\star}\right)+2 \sup _{\tilde{w} \in[0,1]}|H(\tilde{w})-\hat{H}(\tilde{w})| \leq 2 \sup _{\tilde{w} \in[0,1]}|H(\tilde{w})-\hat{H}(\tilde{w})| \rightarrow_{\text {a.s }} 0$ and so, $H\left(\hat{w}^{\star}\right) \rightarrow H\left(w^{\star}\right)$ almost surely, where $w^{\star} \in \Omega_{0}$.

\section{Additional result}

Corollary 1 Let $z$ be a random variable with probability density function bounded by above. Let $\theta_{1}, \ldots, \theta_{N}$ be fixed points in the real line. Suppose that all the assumptions in Theorem 1 hold for each $\theta_{j}$ and the set $\left\{0, F_{S}\left(t ; \theta_{j}\right) / \alpha, 1\right\}$ contains no minimizer of $H\left(\cdot ; \theta_{j}\right)$ for all $j=1, \ldots, N$. Then for each $j$ and $\hat{w}^{\star}\left(\theta_{j}\right)$ minimizer of $\hat{H}\left(; \theta_{j}\right)$ there exists a $w^{\star}\left(\theta_{j}\right)$ minimizer of $H\left(\cdot ; \theta_{j}\right)$ such that

$$
\lim _{n \rightarrow \infty} \operatorname{pr}\left(\left\{\theta_{j}: z \in A_{w^{\star}\left(\theta_{j}\right)}^{S}, j \in\{1, \ldots, N\}\right\}=\left\{\theta_{j}: z \in A_{\hat{w}^{\star}\left(\theta_{j}\right)}^{S}, j \in\{1, \ldots, N\}\right\}\right)=1 .
$$

Proof 2 First, it is immediate from Theorem 1 that (16) holds. Next, let $g$ be the probability 
density function of $g$. Then

$$
\begin{aligned}
& \lim _{n \rightarrow \infty} \operatorname{pr}\left(\left\{\theta_{j}: z \in A_{w^{\star}\left(\theta_{j}\right)}^{S}, j \in\{1, \ldots, N\}\right\}=\left\{\theta_{j}: z \in A_{\hat{w}^{\star}\left(\theta_{j}\right)}^{S}, j \in\{1, \ldots, N\}\right\}\right) \\
& =\lim _{n \rightarrow \infty} \int \operatorname{pr}\left(\left\{\theta_{j}: z \in A_{w^{\star}\left(\theta_{j}\right)}^{S}, j \in\{1, \ldots, N\}\right\}=\left\{\theta_{j}: z \in A_{\hat{w}^{\star}\left(\theta_{j}\right)}^{S}, j \in\{1, \ldots, N\}\right\} \mid z\right) g(z) d z \\
& =\int \lim _{n \rightarrow \infty} \operatorname{pr}\left(\left\{\theta_{j}: z \in A_{w^{\star}\left(\theta_{j}\right)}^{S}, j \in\{1, \ldots, N\}\right\}=\left\{\theta_{j}: z \in A_{\hat{w}^{\star}\left(\theta_{j}\right)}^{S}, j \in\{1, \ldots, N\}\right\} \mid z\right) g(z) d z \\
& =1
\end{aligned}
$$

where the second and third inequalities follow from the dominated convergence theorem and (16).

\section{References}

Yoav Benjamini and Yosef Hochberg. Controlling the false discovery rate: A practical and powerful approach to multiple testing. Journal of the Royal Statistical Society. Series B (Methodological), 57(1):289-300, 1995. ISSN 00359246. URL http://www.jstor.org/stable/ 2346101

Yoav Benjamini and Daniel Yekutieli. False discovery rate-adjusted multiple confidence intervals for selected parameters. Journal of the American Statistical Association, 100(469): 71-81, 2005. doi: 10.1198/016214504000001907. URL https://doi.org/10.1198/ 016214504000001907.

Carlos M. Carvalho, Nicholas G. Polson, and James G. Scott. The horseshoe estimator for sparse signals. Biometrika, 97(2):465-480, 2010. doi: 10.1093/biomet/asq017. URL http://dx. doi.org/10.1093/biomet/asq017

A. P. Dawid. Selection paradoxes of Bayesian inference. Lecture Notes-Monograph Series, 24: 211-220, 1994. ISSN 07492170. URL http: //www.jstor.org/stable/4355805.

B. Efron, R. Tibshirani, J.D. Storey, and V. Tusher. Empirical Bayes analysis of a microarray experiment. Journal of American Statistical Association, 96:1151-60, 2001.

Bradley Efron. Microarrays, empirical Bayes and the two-groups model (with discussion). Statistical Science, 1(23):1-22, 2008. 
W. Fithian, D. Sun, and J. Taylor. Optimal Inference After Model Selection. ArXiv e-prints, October 2014.

Malay Ghosh. Objective priors: An introduction for frequentists. Statistical Science, 26(2): 187-202, 2011. ISSN 08834237. URL http://www.jstor.org/stable/23059978.

Peter Hoff and Chaoyu Yu. Exact adaptive confidence intervals for linear regression coefficients. Electron. J. Statist., 13(1):94-119, 2019. doi: 10.1214/18-EJS1517. URL https://doi .org/ 10.1214/18-EJS1517.

Ryan C. Kelly, Matthew A. Smith, Robert E. Kass, and Tai Sing Lee. Local field potentials indicate network state and account for neuronal response variability. Journal of Computational Neuroscience, 29(3):567-579, Dec 2010. ISSN 1573-6873. doi: 10.1007/s10827-009-0208-9. URL https://doi.org/10.1007/s10827-009-0208-9.

Jason D. Lee, Dennis L. Sun, Yuekai Sun, and Jonathan E. Taylor. Exact post-selection inference, with application to the lasso. Ann. Statist., 44(3):907-927, 062016. doi: 10.1214/15-AOS1371. URL https://doi.org/10.1214/15-AOS1371.

A. Newton. On a nonparametric recursive estimator of the mixing distribution. Sankhyā Ser. A, pages 306-322, 2002.

Trevor Park and George Casella. The Bayesian lasso. Journal of the American Statistical Association, 103(482):681-686, 2008. doi: 10.1198/016214508000000337. URL https: //doi.org/10.1198/016214508000000337.

Nicholas G. Polson and James G. Scott. Shrink globally, act locally: sparse Bayesian regularization and prediction (with discussion). In J. M. Bernardo, M. J. Bayarri, J. O. Berger, A. P. Dawid, D. Heckerman, A. F. M. Smith, and M. West, editors, Proceedings of the 9th Valencia World Meeting on Bayesian Statistics, pages 501-38. Oxford University Press, 2011.

John W Pratt. Length of confidence intervals. Journal of the American Statistical Association, 56(295):549-567, 1961. 
John W. Pratt. Shorter confidence intervals for the mean of a normal distribution with known variance. Ann. Math. Statist., 34(2):574-586, 06 1963. doi: 10.1214/aoms/1177704170. URL https://doi.org/10.1214/aoms/1177704170.

Stephen Reid, Jonathan Taylor, and Robert Tibshirani. Post-selection point and interval estimation of signal sizes in gaussian samples. Canadian Journal of Statistics, 45, 042014.

James G. Scott and James O. Berger. An exploration of aspects of Bayesian multiple testing. Journal of Statistical Planning and Inference, 136(7 SPEC. ISS.):2144-2162, 7 2006. ISSN 0378-3758. doi: 10.1016/j.jspi.2005.08.031.

James G. Scott, Ryan C. Kelly, Matthew A. Smith, Pengcheng Zhou, and Robert E. Kass. False discovery rate regression: An application to neural synchrony detection in primary visual cortex. Journal of the American Statistical Association, 110(510):459-471, 2015. doi: 10.1080/ 01621459.2014.990973. URL https://doi.org/10.1080/01621459.2014.990973.

Matthew A. Smith and Adam Kohn. Spatial and temporal scales of neuronal correlation in primary visual cortex. Journal of Neuroscience, 28(48):12591-12603, 2008. ISSN 0270-6474. doi: 10.1523/JNEUROSCI.2929-08.2008. URL http://www. jneurosci.org/content/28/ $48 / 12591$

Surya T. Tokdar, Ryan Martin, and Jayanta K. Ghosh. Consistency of a recursive estimate of mixing distributions. The Annals of Statistics, 37(5A):2502-2522, 2009. ISSN 00905364. URL http: //www. jstor.org/stable/30243713.

A. W. van der Vaart. Asymptotic Statistics. Cambridge Series in Statistical and Probabilistic Mathematics. Cambridge University Press, 1998. doi: 10.1017/CBO9780511802256.

Abraham Wald. Note on the consistency of the maximum likelihood estimate. Ann. Math. Statist., 20(4):595-601, 12 1949. doi: 10.1214/aoms/1177729952. URL https://doi.org/10.1214/ aoms/1177729952.

Asaf Weinstein, William Fithian, and Yoav Benjamini. Selection adjusted confidence intervals with more power to determine the sign. Journal of the American Statistical Association, 108 
(501):165-176, 2013. doi: 10.1080/01621459.2012.737740. URL https://doi.org/10. $1080 / 01621459.2012 .737740$.

Daniel Yekutieli. Adjusted Bayesian inference for selected parameters. Journal of the Royal Statistical Society: Series B (Statistical Methodology), 74(3):515-541, 2012. ISSN 14679868. doi: 10.1111/j.1467-9868.2011.01016.x. URL http://dx.doi.org/10.1111/j. $1467-9868.2011 .01016 . x$

C Yu and P D Hoff. Adaptive multigroup confidence intervals with constant coverage. Biometrika, 105(2):319-335, 2018. doi: 10.1093/biomet/asy009. URL http://dx.doi.org/ 10.1093/biomet/asy009

Hua Zhong and Ross L. Prentice. Bias-reduced estimators and confidence intervals for odds ratios in genome-wide association studies. Biostatistics, 9(4):621-634, 02 2008. ISSN 14654644. doi: 10.1093/biostatistics/kxn001. URL https://doi.org/10.1093/biostatistics/ kxno01. 